\title{
Percutaneous Transhepatic and Translumbar Sclerotherapy of a Thoracic Duct Cyst: A Case Report
}

\author{
Ibrahim Alrashidi ${ }^{1}$ ji Hoon Shin ${ }^{1}$ \\ ${ }^{1}$ Department of Radiology, University of Ulsan, College of Medicine \\ Asan Medical Center, Songpa-gu, Seoul, Republic of Korea
}

\begin{abstract}
Address for correspondence Ji Hoon Shin, MD, Department of Radiology, University of Ulsan, College of Medicine Asan Medical Center, 86 Asanbyeongwon-gil, Songpa-gu, Seoul 138-736, Republic of Korea (e-mail: jhshin@amc.seoul.kr).
\end{abstract}

\begin{abstract}
Keywords

- sclerotherapy

- thoracic duct cyst

- ethanol

- percutaneous
\end{abstract}

\section{Introduction}

Although uncommon, thoracic duct cysts are lymph-filled collections that may arise from any portion of the thoracic duct. ${ }^{1,2}$ Such cysts may be accompanied with intermittent abdominal pain. ${ }^{1}$

To accurately diagnose and prevent potential complications such as spontaneous or traumatic cyst rupture, surgery and subsequent histological examination are required. ${ }^{2-4}$ Here, we describe percutaneous transhepatic and translumbar sclerotherapy of a thoracic duct cyst located in the right retrocrural and retrocardiac regions, which are difficult to access using conventional surgery. To the best of our knowledge, percutaneous sclerotherapy of a thoracic duct cyst in this challenging location has not been previously described.

\section{Case Presentation}

A 58-year-old male presented with chronic vague abdominal pain lasting for 5 years. The pain was aggravated by respiration and movement. There was no fever, weight loss, or other gastrointestinal symptoms. He had no history of trauma, previous surgery, or congenital abnormalities. Magnetic resonance imaging ( - Fig. 1A) and enhanced computed tomography $(\mathrm{CT})(-$ Fig. 1B) revealed a $15 \times 6 \mathrm{~cm}$, nonenhancing, and multicystic lesion in the right retrocrural and retrocardiac regions, representing a thoracic duct cyst with three compartments and ranging from T5/T6 to T12/L1. There was no ascites, pleural effusion, or lymphedema. After a multidisciplinary discussion with vascular and cardiothoracic teams, it was decided to perform percutaneous sclerotherapy. published online June 4, 2021
DOI https://doi.org/ $10.1055 / \mathrm{s}-0041-1730116$ ISSN 2542-7075
(C) 2021. The Pan Arab Interventional Radiology Society

This is an open access article published by Thieme under the terms of the Creative Commons Attribution-NonDerivative-NonCommercial-License, permitting copying and reproduction so long as the original work is given appropriate credit. Contents may not be used for commercial purposes, or adapted, remixed, transformed or built upon. (https://creativecommons.org/licenses/by-nc-nd/4.0/). Thieme Medical and Scientific Publishers Private Ltd. A-12, Second Floor, Sector -2, NOIDA -201301, India 
The intranodal lymphangiogram was not performed because it was thought that there was no communication with the normal cystic duct due to calcification at the distal part of the cyst. There appeared to be no safe route other than the transhepatic route; therefore, this route was selected to puncture the lower compartment under ultrasound guidance, using a 22-g Chiba needle under local anesthesia. Approximately $50 \mathrm{~mL}$ of yellowish chylous fluid was aspirated (-Fig. 2). Contrast injection in the lower compartment showed a very narrow passage into the middle compartment, and a stiff 0.035 " guidewire barely passed through the connecting region. An 8.5-Fr pigtail catheter was inserted into the middle compartment. Subsequent contrast injection showed minimal contrast passage into the upper compartment through a very narrow neck, but guidewires of various diameters failed to reach into the upper compartment. After excluding any connections with surrounding structures, sclerotherapy was first performed in the middle compartment where the pigtail catheter was located; this was followed by lower compartment sclerotherapy after pulling the catheter so that it was located in the lower compartment. Each compartment was washed three times with $3 \mathrm{~mL}$ of $99 \%$ ethanol to minimize ethanol dilution, followed by indwelling of $11 \mathrm{~mL}$ of $99 \%$ ethanol for 7 minutes, while the patient was positioned from the right to the left lateral decubitus to allow the ethanol to contact the entire cyst wall. Next, the ethanol was aspirated and the catheter removed. The upper compartment had a retrocardiac location and was not accessible from the middle compartment. On prone position, the contrast-filled upper compartment was punctured with a 22-g Chiba needle, using a translumbar approach under cone-beam CT guidance. The needle path passed between the aorta and the spine, followed by the placement of a pigtail catheter over a guidewire, and a similar fluid was aspirated and the contrast injection showed no communication with the surrounding structures. Then the 99\% alcohol sclerotherapy technique was applied similarly as before for the lower and middle compartments.

The patient's pain began to subside over time. A 6-month follow-up CT scan revealed a significant decrease in thoracic duct cyst size $(5 \times 3 \mathrm{~cm})$ ( - Fig. 3$)$, and the patient's pain had disappeared, with no complications related to the procedure during the follow-up period.
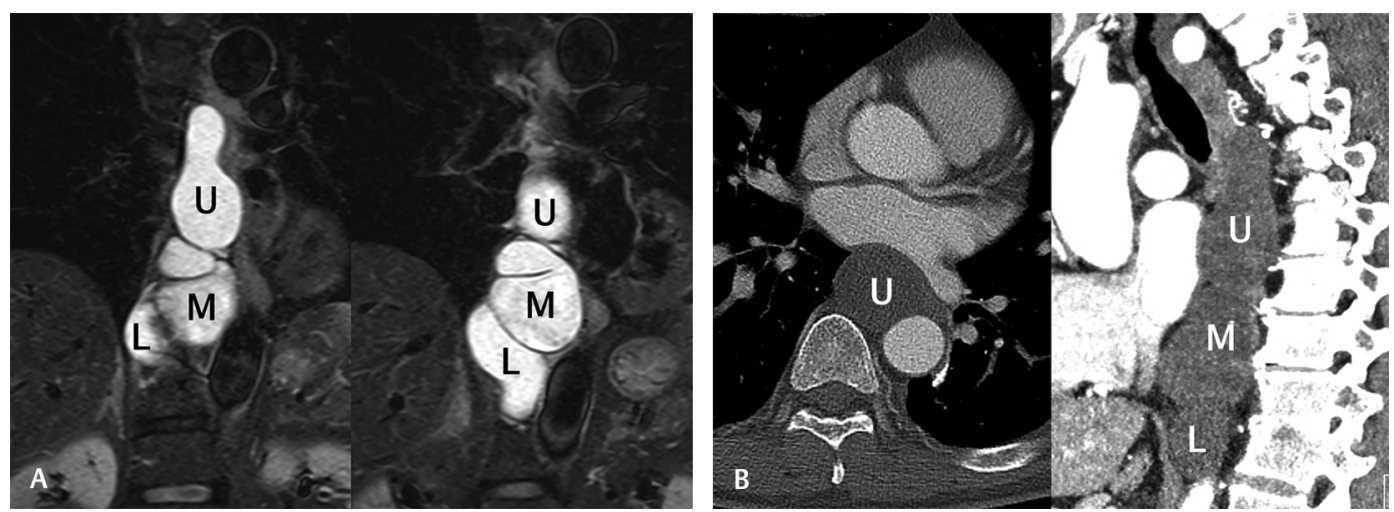

Fig. 1 A 58-year-old male presented with chronic abdominal pain lasting for 5 years. Coronal T2-weighted magnetic resonance (A) and enhanced axial and sagittal computed tomography (B) scans showed a nonenhancing, multicystic lesion in the right retrocrural and retrocardiac regions, representing a thoracic duct cyst with three compartments. L, lower compartment; $\mathrm{M}$, middle compartment; $\mathrm{U}$, upper compartment.
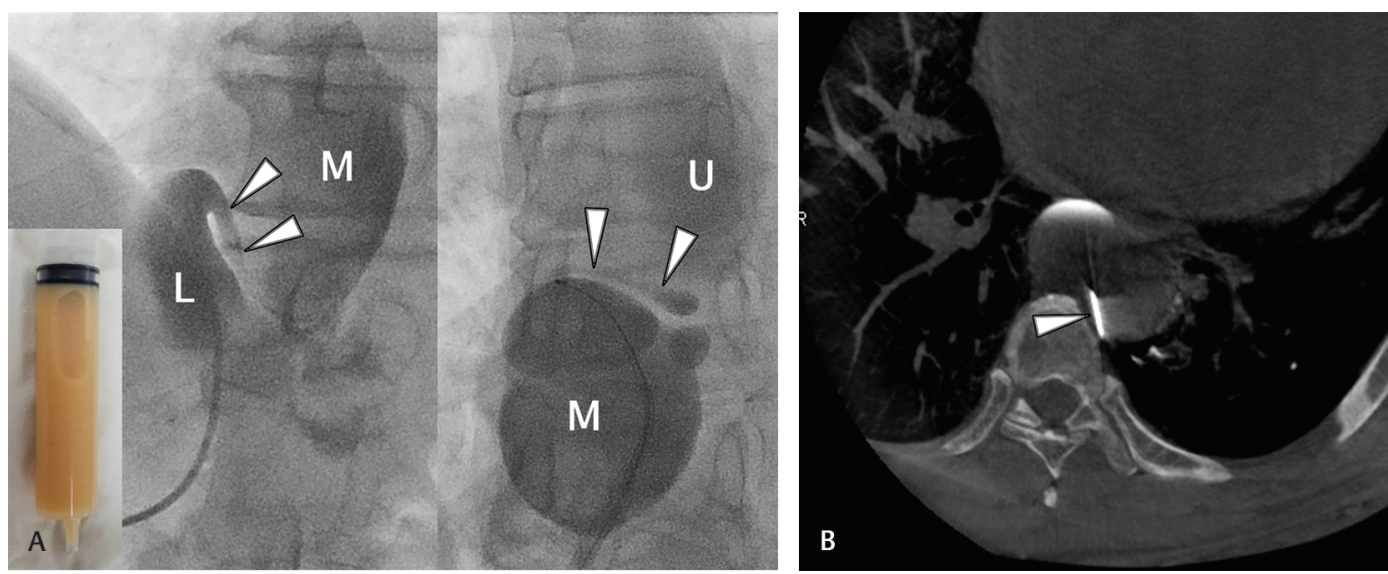

Fig. 2 (A) Serial radiographs of contrast injection at the lower and middle compartments showed limited communication (arrowheads) between the compartments. Note the aspirated chylous fluid (inset). (B) The needle path (arrowhead) between the aorta and the spine using a translumbar approach under cone-beam computed tomography guidance targeting the upper compartment. L, lower compartment; M, middle compartment; $\mathrm{U}$, upper compartment. 

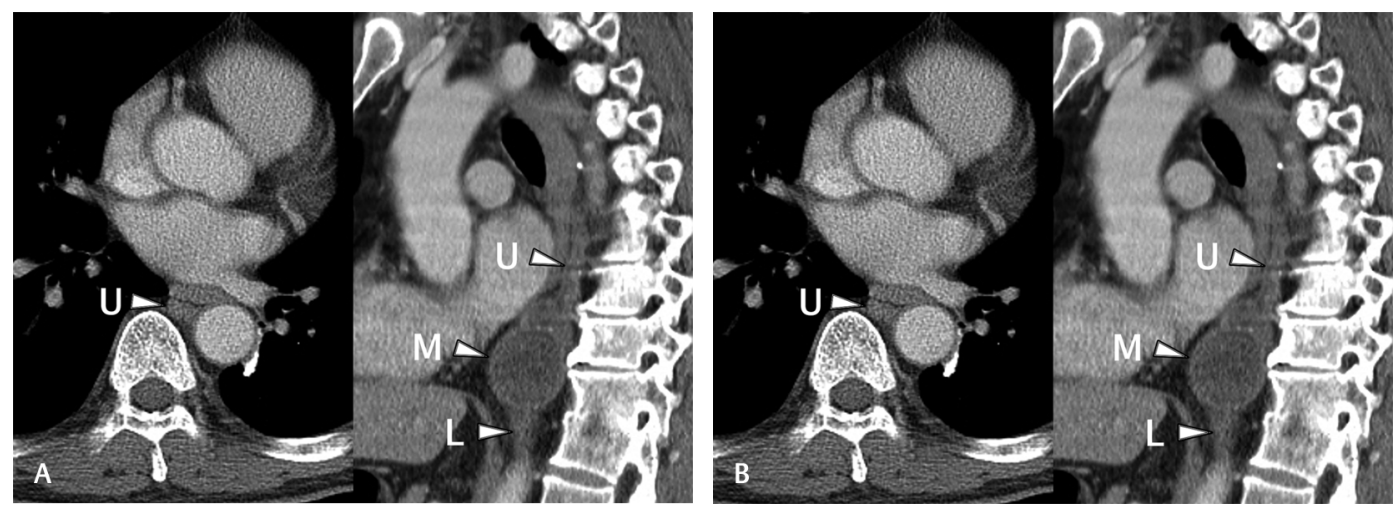

Fig. 3 Six-month follow-up computed tomography scans after sclerotherapy show a decrease in the size of the thoracic duct cyst. L, lower compartment; $\mathrm{M}$, middle compartment; $\mathrm{U}$, upper compartment.

\section{Discussion}

The cyst was considered as a thoracic duct cyst because it was difficult to regard as macrocystic lymphatic malformation because the path of the cyst was very consistent with the thoracic duct. Thoracic duct cysts can be observed anywhere along the course of the thoracic duct, from its origin in the cisterna chyli in the abdomen all the way up to the left part of the neck where the duct is inserted to the junction of the left subclavian vein and internal jugular vein. ${ }^{1,2}$ The pathogenesis of thoracic duct cysts is not clearly known; however, it is assumed to be linked to the congenital or degenerative weakness of the thoracic duct wall, lymphatic flow obstruction, ${ }^{1,5}$ iatrogenic injuries, or blunt traumas. ${ }^{1,6}$ Cysts cause symptoms by applying pressure to adjacent structures. Symptoms such as dysphagia, dyspnea, abdominal pain, spontaneous chylothorax, and supraclavicular or neck masses may develop. ${ }^{1}$

These cysts are usually recommended to be removed surgically. The surgical procedure should be undertaken to validate the diagnosis by histological examination and to avoid possible complications such as spontaneous or traumatic cyst rupture. The most common complication following surgery is chylothorax that needs reoperation or embolization of the thoracic duct. ${ }^{1-4}$

Transcatheter sclerotherapy of cystic lesions has become a safe, effective, minimally invasive treatment option, especially in cases where surgery is difficult and/or results in an incomplete resection, such as this case, where the retrocardiac and retrocrural location of the thoracic duct cyst made it unsuitable for surgical removal. ${ }^{7}$ Among various sclerosing agents, ethanol remains the most cost-effective agent. Ethanol induces denaturation of proteins, cell death, and inflammatory fibrosis after contact with the cyst wall; however, repeated sessions may be necessary to achieve sufficient inflammatory changes and cyst wall scarring. Therefore, contact with the whole cyst wall is essential during treatment., ${ }^{78}$ In adults, the maximum ethanol amount should be limited to $100 \mathrm{~mL}$ to avoid alcohol toxicity, and patient monitoring is important for signs of alcohol toxicity during sclerotherapy. Potential complications of alcohol ablation include pain, as well as bleeding, infection, and injury to adjacent organs. ${ }^{7}$
Translumbar access can be utilized when other endovascular or endoluminal approaches appear infeasible or risky. ${ }^{9,10}$ The translumbar approach is performed under both fluoroscopic and cone-beam CT scanning, and the needle path to the target and possible complications like bleeding or erroneous punctures can be monitored.

\section{Conclusion}

In the retrocardiac and retrocrural areas, this case illustrates successful percutaneous catheter-directed sclerotherapy for a thoracic duct cyst, which would be difficult to remove surgically.

\section{Conflict of Interest}

None.

\section{References}

1 Wan X, Zhou Z. A giant thoracic duct cyst as the cause of abdomen pain: a case report and review of the literature. Ann Thorac Cardiovasc Surg 2015;21(5):487-491

2 Napolitano MA, Mortman KD. Robotic resection of a thoracic duct cyst. J Minim Access Surg 2020;16(4):429-430

3 Kwak MY, Bae CH. Thoracic duct cyst in mediastinum - a case report. Korean J Thorac Cardiovasc Surg 2011;44(1):83-85

4 Abu-Zaid A, Alakhtar AM, Alshamdin FD, Saleh W. Thoracic duct cyst presenting as abdominal pain. Surgery 2018;163(6):1324

5 Mortman KD. Mediastinal thoracic duct cyst. Ann Thorac Surg 2009;88(6):2006-2008

6 Bakst AA. Blind supradiaphragmatic thoracic duct cyst; case report. Ann Surg 1954;140(2):250-253

7 Cheng D, Amin P, Ha TV. Percutaneous sclerotherapy of cystic lesions. Semin Intervent Radiol 2012;29(4):295-300

8 Dool JJ, de Bree R, van den Berg R, Leemans CR. Thoracic duct cyst: sclerotherapy as alternative for surgical treatment. Head Neck 2007;29(3):292-295

9 Lagios K, Karaolanis G, Bazinas T, Perdikides T, Bountouris I. Translumbar infusion of N-butyl cyanoacrylate for the treatment of type II endoleaks. J Vasc Interv Radiol 2018;29(6):826-832

10 Kariya S, Nakatani M, Maruyama T, et al. Central venous access port placement by translumbar approach using angio-CT unit in patients with superior vena cava syndrome. Jpn J Radiol 2018;36(7):450-455 